\title{
Three component time reversal: Focusing vector components using a scalar source
}

\author{
T. J. Ulrich, ${ }^{1, a)}$ Koen Van Den Abeele, ${ }^{2}$ Pierre-Yves Le Bas, ${ }^{1}$ Michele Griffa, ${ }^{3}$ \\ Brian E. Anderson, ${ }^{4}$ and Robert A. Guyer ${ }^{5}$ \\ ${ }_{1}^{1}$ EES-17, Los Alamos National Laboratory, Los Alamos, New Mexico 87545, USA \\ ${ }^{2}$ Catholic University of Leuven, Campus Kortrijk, Kortrijk 8500, Belgium \\ ${ }^{3}$ EMPA, Swiss Federal Laboratories for Materials Testing and Research, Building Technologies Lab, \\ Überlandstrasse 129, 8600 Dübendorf, Switzerland \\ ${ }^{4}$ Department of Physics, Brigham Young University, Provo, Utah 84602, USA \\ ${ }^{5}$ Department of Physics, University of Nevada, Reno, Nevada 89557, USA
}

(Received 2 March 2009; accepted 6 October 2009; published online 2 December 2009)

\begin{abstract}
In acoustics, it is known that, for a given response signal at an arbitrary location, reciprocity and time reversal (TR) can be used to focus high levels of acoustic energy at that position. In solid media, elastic waves generally induce different disturbances in three directions. In this paper, both experimental and numerical wave propagation results for solid materials demonstrate the ability to use a scalar source, a three component detector and the reciprocal TR process to selectively focus each of the different vector components, either individually or collectively. The principle is explained from an analytical point of view. The numerical and experimental study demonstrates excellent temporal and spatial focalization. Applications of the selective vector component focusing can be found in damage imaging techniques using both linear or nonlinear ultrasonic waves. () 2009 American Institute of Physics. [doi:10.1063/1.3259371]
\end{abstract}

\section{INTRODUCTION}

Reciprocity in acoustic and elastic wave propagation is a cornerstone of time reversal (TR) and in using TR as a technique to selectively focus acoustic/elastic energy in a given medium. ${ }^{1-6}$ Typically, in TR studies, the components of the vector wave field are either absent, as in the case of propagation in a fluid, where the wave field is purely scalar, or disregarded, as for most of the solid media, due to difficulties of measuring components other than the normal one (relative to the sample surface). With the advent of TR based techniques in solids, ${ }^{7-17}$ the ability to exploit the vector nature of elastic waves in solids may prove useful in these and other yet to be developed applications. Specifically, it is common in laboratory studies to use scalar sources, i.e., sources having a single vector component in which they displace the media, e.g., compressional transducers. The propagation of this disturbance in a solid converts to different types of wave motion (i.e., shear and compressional) upon encountering scatterers and boundaries. As a result, the wave field detected at some other point on the sample will be composed of motion in each of the three spatial directions, and due to different types of wave motion. Contained herein is a brief analytical description of the procedure for selectively focusing elastic energy along each of the three spatial directions, either individually or collectively, using a scalar source, a three component detector and the TR process (TRP). ${ }^{4}$ We present experimental and numerical simulation wave propagation results proving the feasibility of such a procedure.

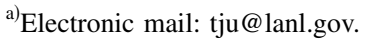

\section{ANALYTICAL DESCRIPTION}

Here, we emphasize the use of reciprocal TR in solids, in the place of standard (a.k.a. classical) TR. ${ }^{6}$ In reciprocal TR, given source and receiver positions $\mathcal{A}$ and $\mathcal{B}$ respectively, the process involves the initial forward propagation of the source from $\mathcal{A}$ to $\mathcal{B}$, followed by the back propagation of the time reversed signals, which in this reciprocal case are also propagating from $\mathcal{A}$ to $\mathcal{B}$. This is in contrast to standard TR in which the back propagation takes place from $\mathcal{B}$ to $\mathcal{A}$. The end result is a focus of energy at the original receiver location (with reciprocal TR) as opposed to focusing upon the original source position, as is the case with standard TR. Spatial reciprocity allows for the interchangeability of positions $\mathcal{A}$ and $\mathcal{B}$ without any consequences on the focused signal. ${ }^{1}$ Small differences do exist, however, and these will be given due attention in the analytical description that follows.

We assume a 3D object of arbitrary geometry with a source location $\mathcal{A}$ and receiver location $\mathcal{B}$, each with their own coordinate system $x_{\mathcal{A}}$ and $x_{\mathcal{B}}$, respectively, as shown in Fig. 1. Here, we will use the standard right hand cartesian coordinate system at both locations such that the unit vectors of $x_{\mathcal{A}}$ are $e_{x}, e_{y}, e_{z}$ and for $x_{\mathcal{B}}$ a prime is added, as in $e_{x^{\prime}}, e_{y^{\prime}}$, $e_{z^{\prime}}$. A source time function $F^{(1)}(t)$ is broadcast from $\mathcal{A}$ with a transducer that produces forces in the direction $e_{\mathcal{A}}=e_{z}$, i.e., the first source function is

$$
S^{(1)}(x, t)=F^{(1)}(t) e_{z} \delta(x-\mathcal{A}) .
$$

The three components of the displacement field are received at $\mathcal{B}$. These displacement fields are in each of the directions defined by the local coordinate system at $x_{\mathcal{B}}$. The detected displacement fields are 


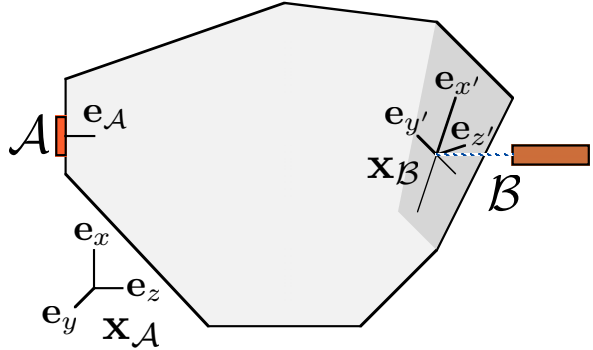

FIG. 1. (Color) Pictoral representation showing a sample with a source transducer capable of emitting a signal unidirectional in the direction $e_{A}$ $=e_{z}$ in the frame $x_{\mathcal{A}}$ at $\mathcal{A}$; and a laser vibrometer receiver measuring the components in the $x_{\mathcal{B}}$ frame at $\mathcal{B}$.

$$
R_{\alpha^{\prime}}^{(1)}(\mathcal{B}, t)=\sum_{\alpha}\left(e_{\alpha^{\prime}} \cdot e_{\alpha}\right) G_{\alpha z}(\mathcal{B} \mid \mathcal{A} ; t) \star F^{(1)}
$$

with " $\star$ " being the standard convolution operator, $\alpha$ representing the $x, y$, and $z$ components and the Green functions are written in terms of the source coordinate system. The three detected displacement fields are separately time reversed and then summed to produce a second source time function

$$
\begin{aligned}
F^{(2)}(t)= & \sum_{\alpha^{\prime}} R_{\alpha^{\prime}}^{(1)}(\mathcal{B},-t)=\sum_{\alpha^{\prime}, \alpha}\left(e_{\alpha^{\prime}} \cdot e_{\alpha}\right) G_{\alpha z}(\mathcal{B} \mid \mathcal{A} ; \\
& -t) \star F^{(1)}(-t) .
\end{aligned}
$$

Alternatively, the sum can be omitted and each component can be used individually or in combination with any other.

The source time function $F^{(2)}(t)$ is broadcast from $\mathcal{A}$ with the transducer that produces forces in the $e_{z}$ direction, i.e., the second source function is

$$
S^{(2)}(x, t)=F^{(2)}(t) e_{z} \delta(x-\mathcal{A}) .
$$

The three components of the displacement field received at $\mathcal{B}$ are

$$
R_{\beta^{\prime}}^{(2)}(\mathcal{B}, t)=\sum_{\beta}\left(e_{\beta^{\prime}} \cdot e_{\beta}\right) G_{\beta z}(\mathcal{B} \mid \mathcal{A} ; t) \star F^{(2)}(t) .
$$

By inserting Eq. (3) into Eq. (5), we have

$$
\begin{aligned}
R_{\beta^{\prime}}^{(2)}(\mathcal{B})= & \sum_{\beta, \alpha, \alpha^{\prime}}\left(e_{\beta^{\prime}} \cdot e_{\beta}\right)\left(e_{\alpha^{\prime}} \cdot e_{\alpha}\right) G_{\beta z}(\mathcal{B} \mid \mathcal{A} ; t) \star G_{\alpha z}(\mathcal{B} \mid \mathcal{A} ; \\
& -t) \star F^{(1)}(-t) .
\end{aligned}
$$

For simplicity, we consider the case in which the primed and unprimed coordinate systems are the same, with the exception of the translation from $\mathcal{A}$ to $\mathcal{B}$. Then, $\alpha^{\prime}=\alpha$ and $\beta^{\prime}$ $=\beta$,

$$
R_{\beta}^{(2)}(\mathcal{B}, t)=\sum_{\alpha} G_{\beta z}(\mathcal{B} \mid \mathcal{A} ; t) \star G_{\alpha z}(\mathcal{B} \mid \mathcal{A} ;-t) \star F^{(1)}(-t) .
$$

Looking at only the $z$ component, Eq. (7) gives us

$$
\begin{aligned}
R_{z}^{(2)}(\mathcal{B}, t)= & G_{z z}(\mathcal{B} \mid \mathcal{A}, t) \star\left[G_{x z}(\mathcal{B} \mid \mathcal{A} ;-t)+G_{y z}(\mathcal{B} \mid \mathcal{A} ;-t)\right. \\
& \left.+G_{z z}(\mathcal{B} \mid \mathcal{A} ;-t)\right] \star F^{(1)}(-t)
\end{aligned}
$$

As the cross terms, e.g., $G_{z z}(\mathcal{B} \mid \mathcal{A} ; t) \star G_{x z}(\mathcal{B} \mid \mathcal{A} ;-t)$, are negligible (i.e., they do not contribute to the TR focus, see the appendix), Eq. (8) reduces to

$$
R_{z}^{(2)}(\mathcal{B}, t) \approx G_{z z}(\mathcal{B} \mid \mathcal{A} ; t) \star G_{z z}(\mathcal{B} \mid \mathcal{A} ;-t) \star F^{(1)}(-t) .
$$

The $x$ and $y$ components can be similarly treated in order to create a TR focus in either the $x$ and/or $y$ motion (separate to or coherent with the focus in $z$ ). For our scalar source (i.e., containing only a $z$ component) the coherent focus in the $x$ and $y$ directions can be taken from Eq. (8) with $\beta=\alpha=x$ or $y$ such that

$$
\begin{aligned}
& R_{x}^{(2)}(\mathcal{B}, t) \approx G_{x z}(\mathcal{B} \mid \mathcal{A} ; t) \star G_{x z}(\mathcal{B} \mid \mathcal{A} ;-t) \star F^{(1)}(-t), \\
& R_{y}^{(2)}(\mathcal{B}, t) \approx G_{y z}(\mathcal{B} \mid \mathcal{A} ; t) \star G_{y z}(\mathcal{B} \mid \mathcal{A} ;-t) \star F^{(1)}(-t) .
\end{aligned}
$$

In any direction of focus, there will remain an incoherent contribution due to the cross terms [e.g., due to $G_{x z}(\mathcal{B} \mid \mathcal{A}$; $-t)$ and $G_{y z}(\mathcal{B} \mid \mathcal{A} ;-t)$ in Eq. (8), the terms not shown in Eqs. (9)-(11)]. While the cross terms in each of the components are sufficiently small, their presence is still detected, as can be seen in both the experimental and modeling results to follow. In short, their effect is a slight increase in the noise floor at the point of focus (i.e., position $\mathcal{B}$ ), as is evident in both the numerical and experimental results that follow. It is worth noting here that this effect, and indeed the existence of these cross terms, is an artifact of performing TR in the reciprocal sense. A true three component elastic TRP in the classical sense would involve the three detected components being broadcast from $\mathcal{B}$ with their original polarizations (i.e., vector component directions), resulting in a reconstruction of the original source with only a single $z$-component polarization. Following a similar analytical process for classical TR as was presented here for reciprocal TR, the analog to Eq. (8) would be

$$
\begin{aligned}
R_{z}^{(2)}(\mathcal{A}, t)= & {\left[G_{z x}(\mathcal{A} \mid \mathcal{B} ;-t) \star G_{x z}(\mathcal{B} \mid \mathcal{A} ; t)+G_{z y}(\mathcal{A} \mid \mathcal{B} ;\right.} \\
& -t) \star G_{y z}(\mathcal{B} \mid \mathcal{A} ; t)+G_{z z}(\mathcal{A} \mid \mathcal{B} ; \\
& \left.-t) \star G_{z z}(\mathcal{B} \mid \mathcal{A} ; t)\right] \star F^{(1)}(-t) .
\end{aligned}
$$

Notice the lack of the cross terms that are present in the reciprocal case [i.e., Eq. (8)]. Throughout the remainder of this paper only the reciprocal case, and its applications, will be considered.

\section{TEMPORAL RESULTS}

\section{A. Modeling}

The first confirmation of the above analysis comes from modeling the wave propagation and comparing the waveforms of the individual components and of their combinations after reciprocal $\mathrm{TR}^{6,11,15}$ has been utilized to focus the elastic energy.

The 3D wave propagation model is based on the staggered grid elastodynamic finite integration technique. ${ }^{18}$ The simulations are performed for a cube sample of fused silicatype material $\left(10 \times 10 \times 10 \mathrm{~cm}^{3}, \quad v_{c} \approx 6000 \mathrm{~m} / \mathrm{s}, \quad v_{s}\right.$ $\left.\approx 2700 \mathrm{~m} / \mathrm{s}, Q \approx 1500, \rho=2500 \mathrm{~kg} / \mathrm{m}^{3}\right)$. As the first source $S^{(1)}(t)$, a short pulse of $200 \mathrm{kHz}$ frequency is considered 


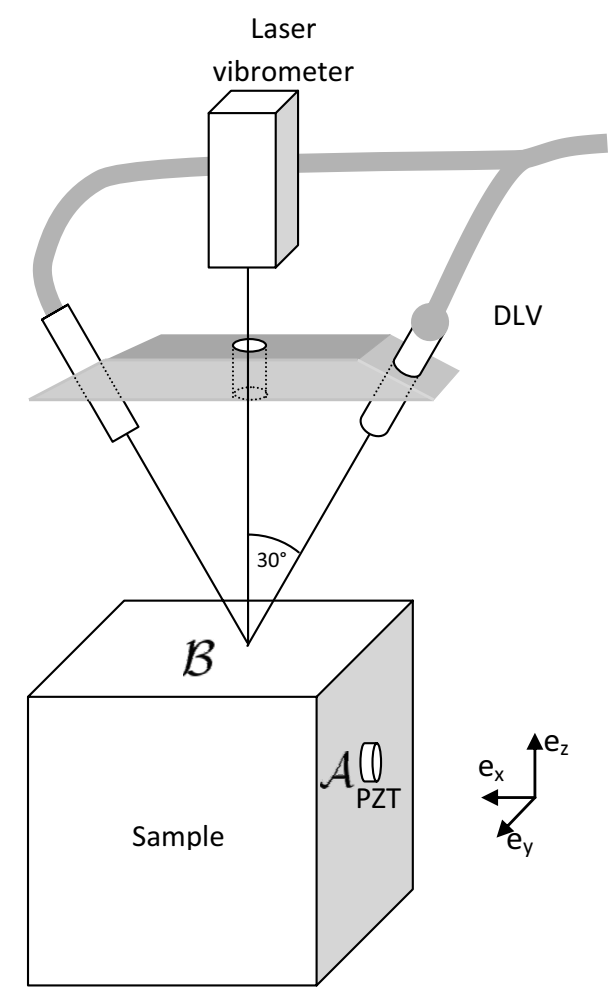

FIG. 2. Experimental setup. The DLV used to obtain the in-plane components is mounted on a rotational stage in order to measure the $x$ and $y$ components individually. The DLV and normal LV are focused coincidently and synced to obtain in-plane and out-of-plane components simultaneously. For the experiments reported here $x_{\mathcal{A}}=x_{\mathcal{B}}$, the unit vectors for which are shown.

which is excited on the $(y-z)$ plane, centered at position $\mathcal{A}$ $(x=0 \mathrm{~cm}, y=4.2 \mathrm{~cm}, z=4.8 \mathrm{~cm})$. An in-phase signal with amplitudes modified by a double Gaussian envelope in space (i.e., Gaussian shaped in both $x$ and $y$ directions) is considered around the center position within a radius of $6 \mathrm{~mm}$. The motion of the emitter is assumed to be out of plane ( $x$-direction). The three particle velocity components $\left[v_{x}\right.$ $=R_{x}^{(1)}(t), v_{y}=R_{y}^{(1)}(t)$ and $\left.v_{z}=R_{z}^{(1)}(t)\right]$ at a receiving location $\mathcal{B}$ in the $(x-y)$ plane at $\mathcal{B}(x=3.7 \mathrm{~cm}, y=5.8 \mathrm{~cm}, z=10 \mathrm{~cm})$ are calculated for $0 \leq t \leq 3.4 \mathrm{~ms}$. The source and receiver positions $\mathcal{A}$ and $\mathcal{B}$ can be seen in the experimental setup in Fig. 2 , and were the same for the numerical model. In this configuration, the out-of-plane motion at the receiver is the $z$-component, the other two components represent the inplane motions. Subsequently, the signals are time reversed. The time reversed signals, individually or summed, are then broadcast again from the emitter location (with motion in $x$-direction) and the three component waveforms $\left[R_{x}^{(2)}(t)\right.$, $R_{y}^{(2)}(t)$, and $\left.R_{z}^{(2)}(t)\right]$ at the receiving location $\mathcal{B}$ are calculated.

Figure 3 provides an overview of the time signals at the receiver for each of the three components when only $v_{x}, v_{y}$, $v_{z}$, or combinations such as $v_{x}+v_{y}, v_{x}+v_{z}, v_{y}+v_{z}$, and $v_{x}$ $+v_{y}+v_{z}$, are considered in the rebroadcast signal. In all cases, it is clear that the TR procedure allows the scalar information which is supplied to the source to be separated into the correct vector components. For instance, when $v_{x}$ $+v_{z}$ is considered as input signal, the time reversed signal will redistribute and focus the energy in the $x$ and $z$ direction at the receiving point, without focusing at the $y$-direction.

\section{B. Experiment}

The above procedure can be realized entirely in the laboratory. In measuring elastic wave propagation, the common practice is to use the noncontact technique of laser vibrometry to obtain the displacement or velocity wave field; again, typically only the component normal to the surface is used. The technology exists to also measure the in-plane components of the vector wave field using 3D laser vibrometers, such as those commercially available from Polytec Inc. Here, the measurements of the three velocity vector components $v_{x}, v_{y}$, and $v_{z}$ were made using two Polytec laser vibrometers, the OFV 303 (3001 controller, VD- $02,25 \mathrm{~mm} / \mathrm{s} / \mathrm{V})$ for the normal $(z)$ component and the OFV 552 (5000 controller, VD- $02,25 \mathrm{~mm} / \mathrm{s} / \mathrm{V})$ fiber-optic differential laser vibrometer (DLV) for the in-plane ( $x$ and $y)$ components. To obtain the in-plane components using the DLV the two fiber-optic laser heads were placed at $\pm 30^{\circ}$ from vertical such that the normal component is eliminated through subtraction, thus leaving only the in-plane component (Fig. 2). ${ }^{19}$ The orientation pictured in Fig. 2 provides a single component of the inplane response (the $x$ component as pictured). In order to obtain the orthogonal component $y$, the apparatus is rotated $90^{\circ}$. The rotation is done using a rotational stage (SR50CC stage \& ESP300 controller from Newport) having a precision of $1 \times 10^{-3}$ degrees and an accuracy of $0.035^{\circ}$.

The sample in which the selective component focusing studies were conducted is a fused silica glass block having identical dimensions and physical parameters as previously defined in the model. To transmit the elastic energy into the sample a piezoelectric disk $\left(d_{33}\right.$ polarized PZT-5, diameter $d=12 \mathrm{~mm}$, thickness $h=2 \mathrm{~mm}$ ) bonded to a surface in the $y-z$ plane, orthogonal to that where the TR focused signals were measured $(x-y$ plane). As the PZT source is only capable of one type of motion (e.g., compressional, here $e_{x}$ ), regardless of the waveform sent to excite it, the transducer is here referred to as a scalar source.

The TR focusing employs reciprocal $\mathrm{TR}^{6}$ in order to use the three component signals measured using the laser vibrometers to create virtual sources of one or more components of motion. This is achieved by emitting a source function $S^{(1)}(t)$, e.g., a $200 \mathrm{kHz}$ tone-burst (duration $\tau=50 \mu \mathrm{s}$ ), from the PZT disk at position $\mathcal{A}$ and recording the three components of the response $R_{x}^{(1)}(t), R_{y}^{(1)}(t)$, and $R_{z}^{(1)}(t)$ at a position $\mathcal{B}$, using the laser vibrometers as shown in Fig. 2. The virtual sources are then created by broadcasting the TR versions of those three components, individually or in combination. The results of this process can be seen in Fig. 4 illustrating the effectiveness of selectively focusing single or multiple components to create virtual sources using reciprocal TR and verifying the results seen from the model in Fig. 3.

\section{SPATIAL RESULTS}

While the temporal reconstruction of energy at the focal time is one feature of TR focusing, it only indicates a focusing in time. TR also focuses the energy spatially in a region defined by the dominant wavelength of the propagating waves. The burden of proof now is to show that, indeed, the 


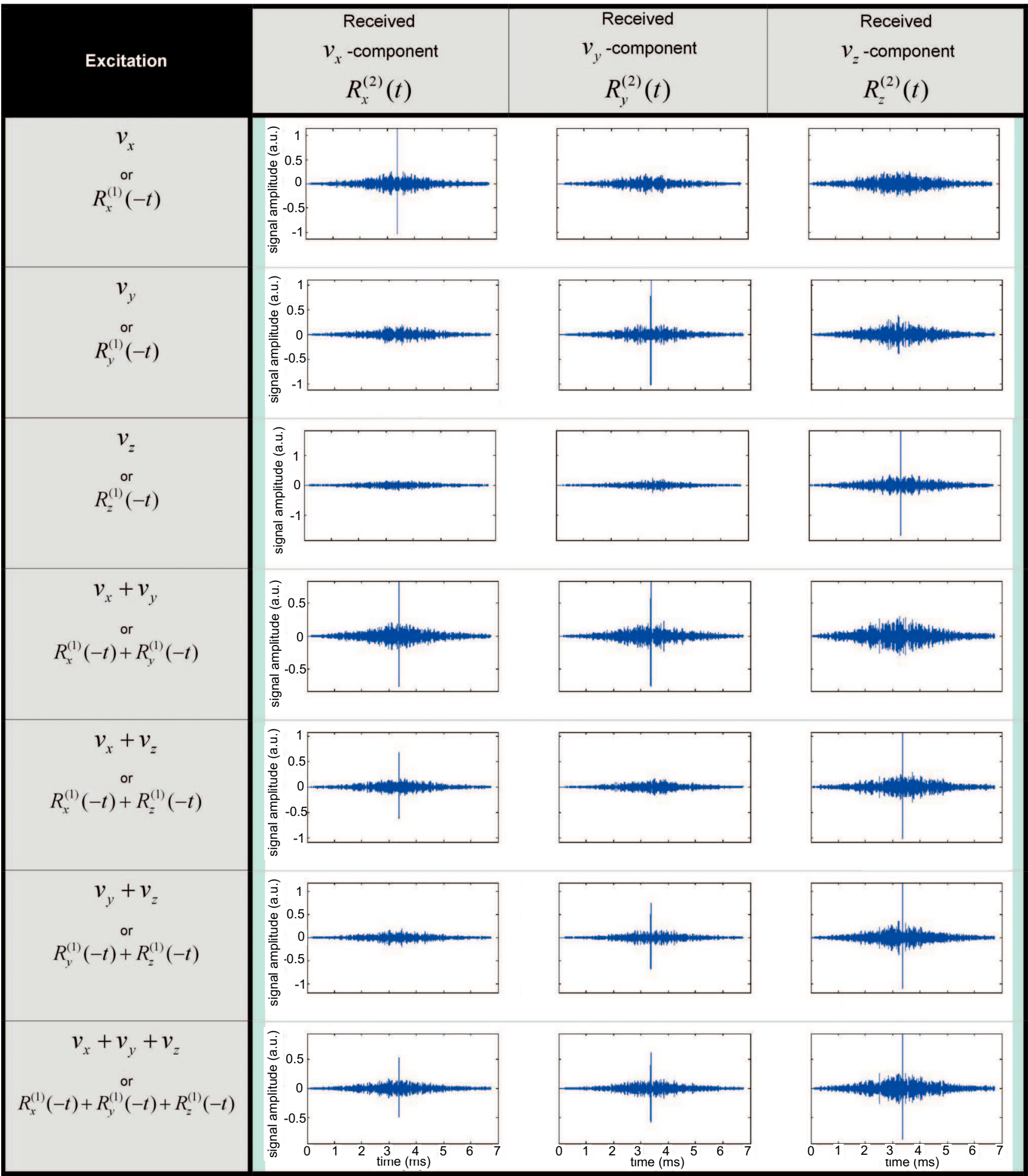

FIG. 3. (Color) Numerical simulations of the selective focusing after reciprocal TR of individual particle velocity components and of various combinations. The simulations are performed for a cube of fused silica, and for $x_{\mathcal{A}}=x_{\mathcal{B}}$ with excitation in the $(y-z)$ plane, reception in the $(x-y)$ plane. Left column specifies the scalar input information supplied to the source for rebroadcasting at position $\mathcal{A}$. Right column displays the subsequent recording of the three particle velocity components at location $\mathcal{B}$.

TRP results in spatial focusing of energy in only the selected component directions. This can be seen both in experimental and modeling results shown in Figs. 5-7.

Figure 5 illustrates the ability of TR to spatially focus any of the three components of motion detected during the forward propagation. Here, each component was focused by creating the source function $S^{(2)}$ from Eq. (4) with $F^{(2)}$
$=\hat{R}_{x}^{(1)}+\hat{R}_{y}^{(1)}+\hat{R}_{z}^{(1)}$, and broadcasting this $S^{(2)}$ from the transducer located at $\mathcal{A}$. The maximum amplitude of the time signal for all three velocity components is shown in a 40 by $40 \mathrm{~mm}$ area of the $(x-y)$ plane around the receiver location $\mathcal{B}$. The color scales are normalized to the maximum amplitude in each respective component. We note a near identical focal 

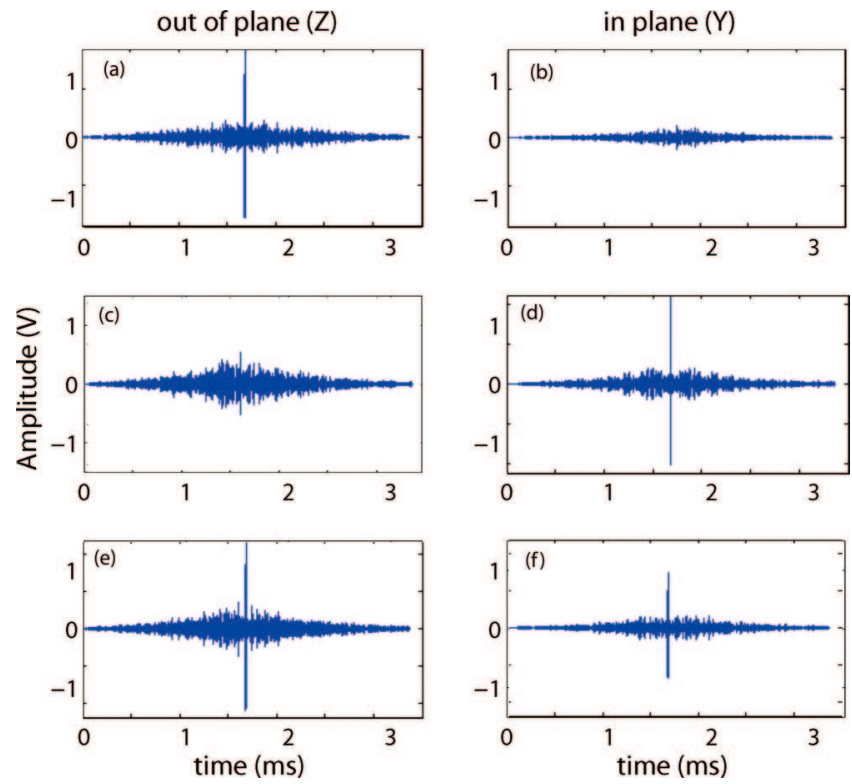

FIG. 4. (Color) Experimentally obtained signals during the reciprocal TR focusing process. (a) \& (b) Respectively, the normal $(z)$ and in-plane $(y)$ component signals recorded during the broadcast of $R_{z}^{(1)}(-t)$; note the high amplitude focusing that occurs in the $z$ component and is absent in the $y$ (also absent in $x$, not shown). (c) \& (d) The $z$ and $y$ component signals recorded during the broadcast of $R_{y}^{(1)}(-t)$; note the focusing now seen in the $y$ component, not in the $z$ or $x$ (not shown). (e) \& (f) The $z$ and $y$ component signals recorded during the broadcast of $R_{z}^{(1)}(-t)+R_{y}^{(1)}(-t)$; note the focusing now seen in both components. The reduced amplitude of each focus is due to the conservation of energy in that one transducer is only capable of emitting a finite amount of energy into the system.

structure for each component, indicating a highly effective localization of energy in each of the three directions.

When focusing a single component, such as the $z$ (out of plane) component, there are a few notable features. With the $z$ component focus there is a central point of focus at $(20,20)$ $\mathrm{mm}$, Fig. 6(c). In the $x$ and $y$ components there is a symme-
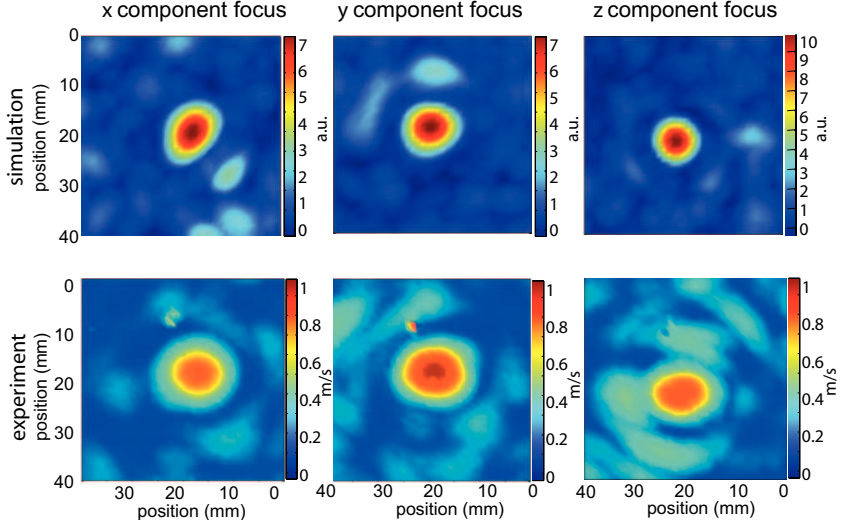

FIG. 5. (Color) Three component focusing with $R_{x}^{(1)}(-t)+R_{y}^{(1)}(-t)+R_{x}^{(1)}(-t)$. Model (top row) and experimental (bottom row) results. Magnitude of the three velocity components of motion $R_{x}^{(2)}(t)=v_{x}, R_{y}^{(2)}(t)=v_{y}$ and $R_{z}^{(2)}(t)=v_{z}$. The color scales are normalized to the maximum amplitude in each respective component. Note the near identical focal structure for each component.

try about the $y=20 \mathrm{~mm}$ and $x=20 \mathrm{~mm}$ lines, Figs. 6(a) and $6(\mathrm{~b})$, respectively. This symmetry indicates a dipole structure in the in-plane components due to the out of plane focusing. This is not surprising in light of Poisson's effect. If one imagines pulling on a string attached to a point on a membrane, the resulting motion in the plane of the membrane would be to move the membrane toward the string; thus in a Cartesian coordinate system the motion would appear to be in either the positive or negative $x$ and $y$ directions depending on the relative location of the attached string. This is exactly what is seen in the amplitudes of the three components in Fig. 6.

By looking at the out of plane and in-plane magnitudes (i.e, $\left|v_{z}\right|$ and $\sqrt{v_{x}^{2}+v_{y}^{2}}$, respectively), a ring can be seen in the in-plane motion that surrounds the focal location (Fig. 7). This is another indication of Poisson's effect, now seen in the
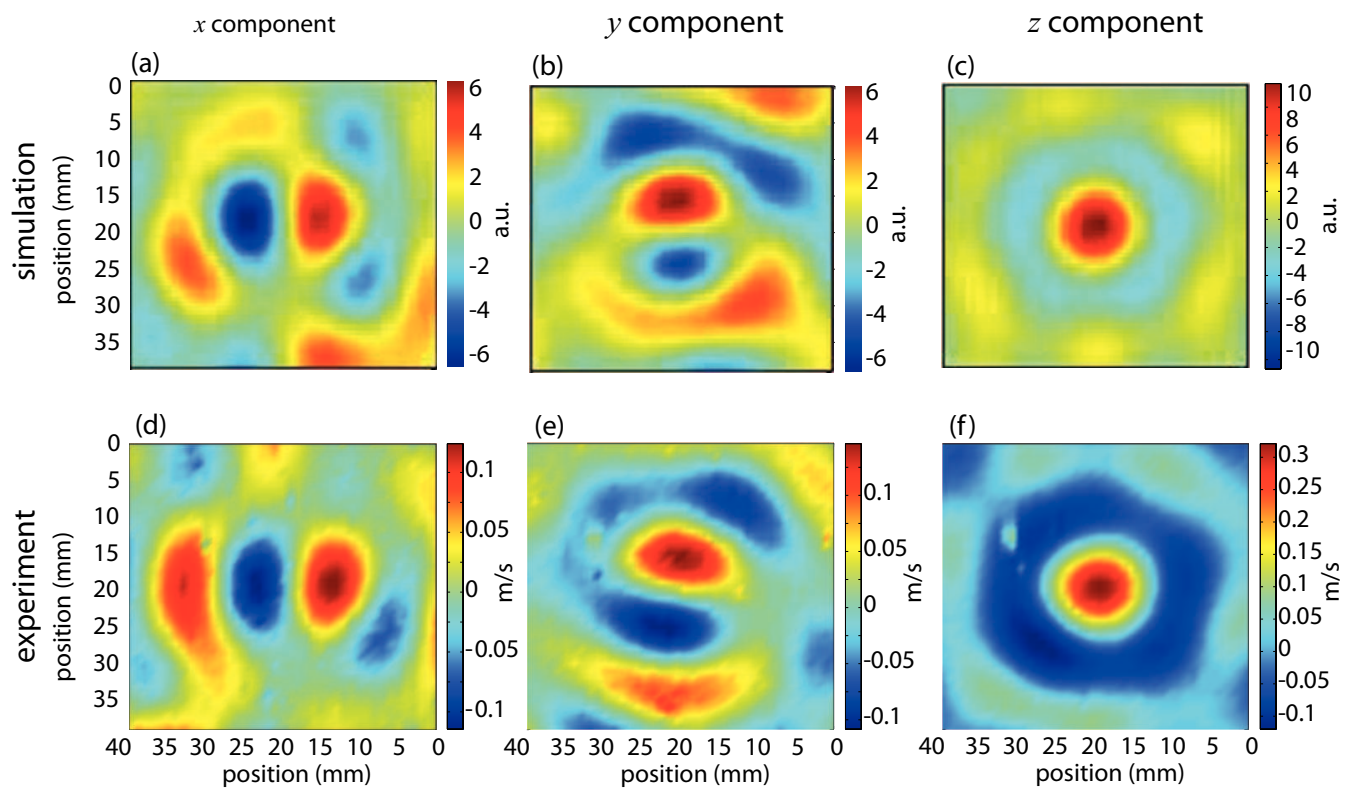

\section{(e)}
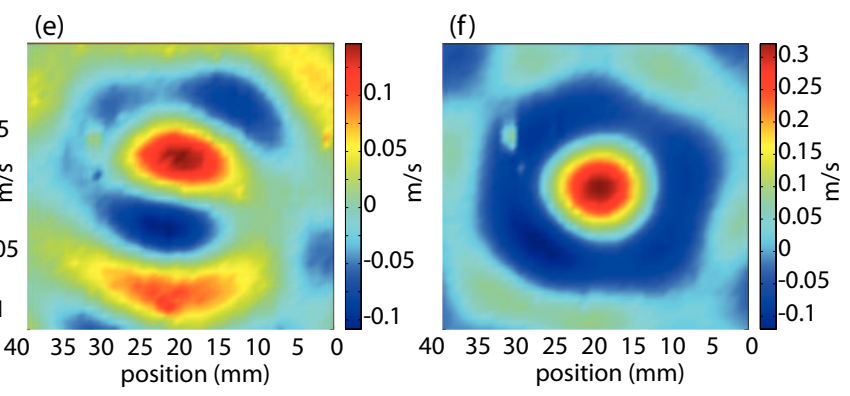

FIG. 6. (Color) Single component $(z)$ focusing with $R_{z}^{(1)}(-t)$. Model (top row) and experimental (bottom row) results. Three velocity components of motion $R_{x}^{(2)}(t)=v_{x}, R_{y}^{(2)}(t)=v_{y}$ and $R_{z}^{(2)}(t)=v_{z}$. The color scales are normalized to the maximum amplitude in each respective component to illustrate the full structure of the vector wave field. Note the in-plane dipole motion seen in the $v_{x}$ and $v_{y}$ components around the focal location due to the Poisson effect. 
(a)
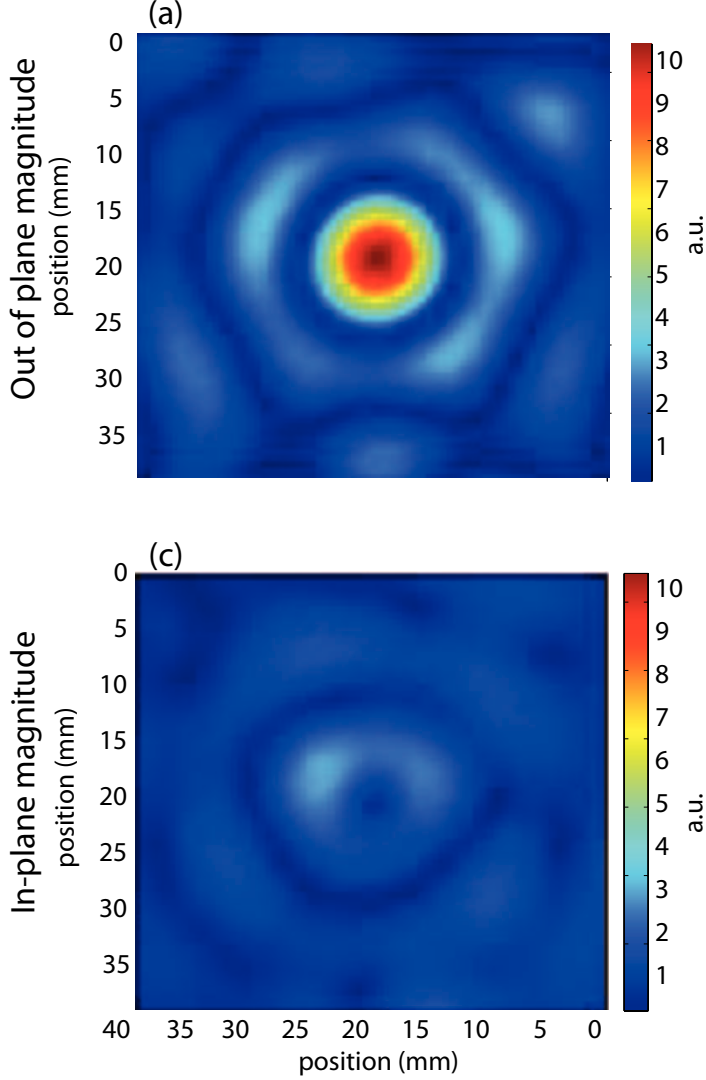

(b)

Experiment

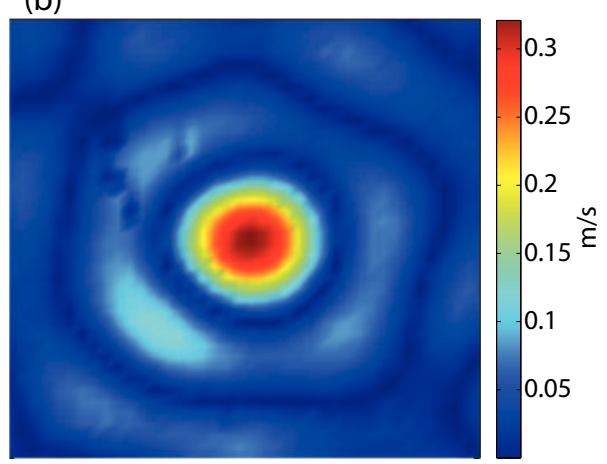

(d)

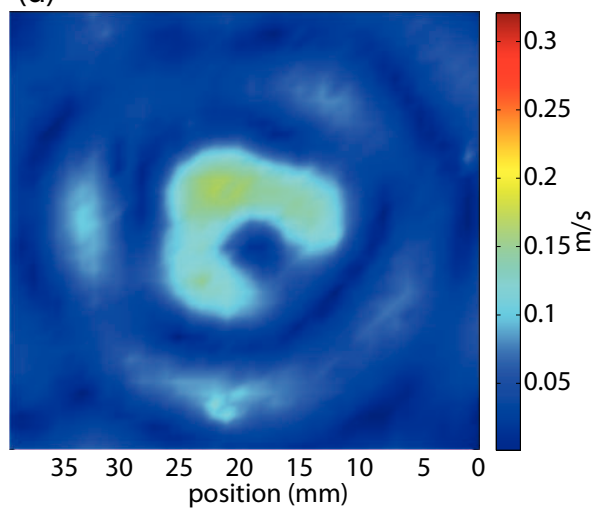

FIG. 7. (Color) Single component $(z)$ focusing with $R_{z}^{(1)}(-t)$. Model $[(a) \&(c)]$ and experimental $[($ b) \& (d)] results. (a) \& (b) Out of plane and (c) \& (d) in-plane magnitudes, i.e., $\left|v_{z}\right|$ and $\sqrt{v_{x}^{2}+v_{y}^{2}}$, respectively. Each column uses the same color scale to accurately depict the relative amplitudes of the in-plane and out of plane components at the time of focus. The Poisson effect is manifest in the in-plane components around the focal location, however, these amplitudes, while higher than the background, are still small with respect to the focal amplitude along $z$ (out of plane).

entire plane at once, as opposed to the individual $x$ and $y$ components. Three notable features are present in this "ring." First, there exists a null at the center, i.e., the point of the $z$ focus, indicating that at that point there is only one component of motion, which was the original intention. Second, the largest amplitudes in-plane are still relatively small compared to the amplitude of the focus in the $z$ direction. And third, the ring is incomplete, meaning it doesn't appear to be closed. This is more of an indication that the TRP did not completely reconstruct the wave field over the entire sample, however at the point of focus the recreation is sufficient. As this is a result from a single channel, it is not that surprising to find incomplete reconstructions of the wave field. Presumably additional channels in the TR mirror would improve the wave field reconstruction.

\section{CONCLUSION}

This study provides an analytical explanation accompanied by both numerical and experimental supporting evidence of the ability of TR to selectively (or collectively) focus the individual components of motion $x, y$, and $z$. This is an important point to be made for anyone interested in creating complex sources in the laboratory, for example, to excite a specific component of motion at an interface, to study earthquake type sources (i.e., double couple sources), or possibly to develop nondestructive evaluation tools by probing structures with precisely defined motions. At this time, studies of interfaces and cracks has begun to utilize the different components of motion in efforts to interrogate the mechanical response of the structures. Seismologists have already employed this aspect of focusing individual displacement components using TR in numerical models ${ }^{16}$ in order to locate glacial earthquakes and determine the direction of the glacier's motion. Undoubtedly more applications exist, including the potential to measure the local dynamic Poisson's ratio.

\section{ACKNOWLEDGMENTS}

The work was supported by Institutional Support (Campaign 8 and LDRD) at the Los Alamos National Laboratory. The authors are grateful for discussions with colleagues Paul Johnson and Carene Larmat on the topics of Time Reversal and reciprocity. Koen Van Den Abeele gratefully acknowledges the support of the European FP6 Grant AERONEWS (Grant No. AST-CT-2003-502927), the Flemish Fund for Scientific Research (Grant Nos. G.0206.02, G.0554.06, and G.0443.07), the Research Council of the Katholieke Universiteit Leuven (Grant No. OT/07/051, CIF1), and the institutional support of the Los Alamos National Laboratory. Special thanks are given to Francesco Simonetti of Imperial College, for discussions on methods of measuring in-plane motion. 


\section{APPENDIX: RANDOM PHASE APPROXIMATION}

In order to address what we refer to the as the crossterms of Eq. (8), specifically as to the assumption that they are negligible, we will employ the random phase approximation. This appendix contains a modest formalism for the situation, i.e., reciprocal TR, described in this paper and the effect that the random phase approximation has on the terms in Eq. (8). This assumption has been utilized in other aspects of TR, for example by Derode et al. in studying the limits of time-reversal focusing through multiple scattering. ${ }^{20}$

The initial broadcast is in direction $\alpha$ from a transducer at $\mathcal{A}$. This broadcast is received at $\mathcal{B}$ where the $\nu$ component of displacement is detected $(\nu=x, y, z$ and $\alpha$ is one of $x, y, z)$. The signal received at $\mathcal{B}$ is

$$
r_{\nu}(t)=\int d t^{\prime \prime} G_{\nu \alpha}\left(\mathcal{B}, t \mid \mathcal{A}, t^{\prime \prime}\right) S_{0}\left(t^{\prime \prime}\right)
$$

This signal is time reversed about the time $T$ to form $r_{\nu}(T$ $-t$ ) and then rebroadcast from $\mathcal{A}$ in direction $\alpha$. The resulting signal is

$$
R_{\nu \nu^{\prime}}(t)=\int d t^{\prime} G_{\nu^{\prime} \alpha}\left(\mathcal{B}, t \mid \mathcal{A}, t^{\prime}\right) r_{\nu}\left(T-t^{\prime}\right)
$$

or

$$
\begin{aligned}
R_{\nu \nu^{\prime}}= & \int d t^{\prime} \int d t^{\prime \prime} G_{\nu^{\prime} \alpha}\left(\mathcal{B}, t \mid \mathcal{A}, t^{\prime}\right) G_{\nu \alpha}(\mathcal{B}, T \\
& \left.-t^{\prime} \mid \mathcal{A}, t^{\prime \prime}\right) S_{0}\left(t^{\prime \prime}\right)
\end{aligned}
$$

Assume that the Green function $G_{\nu \mu}\left(\mathcal{B}, t \mid \mathcal{A}, t^{\prime}\right)$ can be built up as the sum of broadcasts over a family of paths, i.e.,

$$
G_{\nu \mu}\left(\mathcal{B}, t \mid \mathcal{A}, t^{\prime}\right)=\sum_{n} a_{\nu \mu}(n) \delta\left(t-t^{\prime}-\tau_{n}\right),
$$

where the path time $\tau_{n}$ and amplitudes $a_{\nu \mu}(n)$ depend on $\mathcal{A}$ and $\mathcal{B}$. Substitution of this form for $G$ into Eq. (A3) leads to

$$
R_{\nu \nu^{\prime}}(t)=\sum_{n} \sum_{n^{\prime}} a_{\nu^{\prime} \alpha}\left(n^{\prime}\right) a_{\nu \alpha}(n) S_{0}\left(T-t-\tau_{n}+\tau_{n^{\prime}}\right) .
$$

The leading contribution of $R_{\nu \nu^{\prime}}(t)$ is the diagonal term, $n^{\prime}$ $=n$, for which

$$
R_{\nu^{\prime} \nu}(t)=A_{\nu^{\prime} \nu} S_{0}(T-t),
$$

where

$$
A_{\nu^{\prime} \nu}=\sum_{n} a_{\nu^{\prime} \alpha}(n) a_{\nu \alpha}(n)=\left\langle a_{\nu^{\prime} \alpha} a_{\nu \alpha}\right\rangle .
$$

In the random phase approximation,

$$
A_{\nu^{\prime} \nu}=\delta_{\nu^{\prime} \nu}\left\langle a_{\nu \alpha}^{2}\right\rangle
$$

or

$$
R_{\nu^{\prime} \nu}(t)=\delta_{\nu^{\prime} \nu}\left\langle a_{\nu \alpha}^{2}\right\rangle S_{0}(T-t) .
$$

The random phase approximation consists of the assertion that while $a_{z y} a_{z y}$ is necessarily greater than 0 (consequently $\left\langle a_{z y}^{2}\right\rangle$ greater than 0) there is no reason, on average, for the correlation of the sign of $a_{z y}$ and the sign of $a_{z y}$. Thus, $\left\langle a_{z y} a_{x y}\right\rangle \approx 0$ resulting in the cross-terms of Eq. (8) being negligible and therefore ignored in Sec. II.

${ }^{1}$ J. D. Achenback, Reciprocity in Elastodynamics (Cambridge University Press, Cambridge, 2003).

${ }^{2}$ I. Tolstoy and C. Clay, Ocean Acoustics. Theory and Experiment in Underwater Sound (McGraw-Hill, New York, 1966), Chap. 7, pp. 243-268.

${ }^{3}$ M. Fink, D. Cassereau, A. Derode, C. Prada, P. Roux, M. Tanter, J.-L. Thomas, and F. Wu, Rep. Prog. Phys. 63, 1933 (2000).

${ }^{4}$ M. Tanter, J.-L. Thomas, and M. Fink, J. Acoust. Soc. Am. 108, 223 (2000).

${ }^{5}$ J. de Rosny and M. Fink, Phys. Rev. Lett. 89, 124301 (2002).

${ }^{6}$ B. E. Anderson, C. Larmat, M. Griffa, T. Ulrich, and P. A. Johnson, Acoust. Today 4, 5 (2008).

${ }^{7}$ C. Draeger, D. Casserau, and M. Fink, Appl. Phys. Lett. 72, 1567 (1998).

${ }^{8}$ C. Draeger D. casserau, and M. Fink, J. Acoust. Soc. Am. 102, 1289 (1997).

${ }^{9}$ A. Sutin, J. A. TenCate, and P. A. Johnson, J. Acoust. Soc. Am. 116, 2779 (2004).

${ }^{10}$ T. Ulrich, P. A. Johnson, and A. Sutin, J. Acoust. Soc. Am. 119, 1514 (2006).

${ }^{11}$ A. Sutin, B. Libbey, V. Kurtenoks, D. Fenneman, and A. Sarvazyan, Proc. SPIE 6217, 1 (2006).

${ }^{12}$ C. Larmat, J.-P. Montagner, M. Fink, Y. Capdeville, A. Tourin, and E. Clevede, Geophys. Res. Lett. 33, L19312 (2006).

${ }^{13}$ A. S. Gliozzi, M. Griffa, and M. Scalerandi, J. Acoust. Soc. Am. 120, 2506 (2006).

${ }^{14}$ T. Ulrich, P. A. Johnson, and R. A. Guyer, Phys. Rev. Lett. 98, 104301 (2007).

${ }^{15}$ T. Ulrich, A. M. Sutin, T. Claytor, P. Papin, P.-Y. Le Bas, and J. A. TenCate, Appl. Phys. Lett. 93, 151914 (2008).

${ }^{16}$ C. Larmat, J. Tromp, Q. Liu, and J.-P. Montagner, J. Geophys. Res. 113, B09314 (2008)

${ }^{17}$ C. Draeger and M. Fink, Phys. Rev. Lett. 79, 407 (1997).

${ }^{18}$ P. Fellinger, R. Marklein, K. J. Langenberg, and S. Klaholz, Wave Motion 21, 47 (1995).

${ }^{19}$ F. Simonetti and P. Cawley, J. Acoust. Soc. Am. 114, 158 (2003).

${ }^{20}$ A. Derode, A. Tourin, and M. Fink, J. Acoust. Soc. Am. 107, 2987 (2000). 